\title{
Research dedusting efficiency of the inertial hub with adjustable parameters
}

\author{
Rashid Sharapov ${ }^{1,}$, , Pavel Kapyrin ${ }^{1}$, Svetlana Lozovaya ${ }^{2}$, Valentina Yadykina $^{2}$, Aleksandr \\ Agarkov $^{2}$ \\ ${ }^{1}$ Moscow State University of Civil Engineering, Moscow, Russia \\ ${ }^{2}$ Belgorod State Technological University named after V.G. Shukhov, Belgorod, Russia
}

\begin{abstract}
Production of construction materials every year production increases, thus increasing emissions pollutants into the atmosphere subjected to cleaning. At the enterprises of building materials production equipment becoming outdated both morally and physically exhausted their useful life. Technological design standards in the operation of purification equipment has undergone a number of changes. There are a large number of works devoted to the theory of cyclone separation. They examined calculation method and to determine the minimum size of particles is separated completely from the air stream in a cyclone. In theory, the high efficiency cyclones are relatively small separation of particles from the air stream. The experiments show that certain fractions of full capture occurs from the cyclones and out relatively large particles. Designed dust device hubs. They capture dust particles with low specific density and smaller than 5-10 microns. Rational use of hubs is achieved in the aspiration systems high performance. They are placed in front of or between the filter and the cyclone filter. Hub much reduces the amount of dust-laden air directed onto a thin cleaning. This reduces energy consumption for manufacturing as a whole, as well as, due to a possible decrease in performance fine filters, reducing their initial cost, metal consumption, maintenance costs and reduce the area occupied by them.
\end{abstract}

\section{Introduction}

The rapid growth of industrial production, the accumulation of huge amounts of waste, significant energy consumption in various industries contributes pollution of our planet [16].

The catastrophic increase in air pollution due to the sharp disparity between the development of industrial production and equipment cleaning, in particular of air dust, which has not been able to solve the problems posed to the industry. In the future, dedusting equipment has evolved significantly, and the growth of air pollution in some regions has been suspended.

\footnotetext{
Corresponding author: ptdm zavkaf@mail.ru
} 
At the same time successes are local, and air pollution is increasing. This is indirectly saying and reducing life expectancy in selected industrial areas in different countries and a global average temperature increase of our planet [7].

One of the most important tasks for the prevention of the consequences of air pollution is the use of modern dust removal equipment in all process stages of technological processes related to dust emission. This equipment can be used not only in the last stage dedusting process, but also in the early stages, reducing the load on the finishing dedusting.

The aim of this work is the development of inertial dust hub, providing more efficient clearing of coarse dusty process gases above $90 \%$ while reducing its hydraulic resistance.

\section{Development of the concept of inertial dust hub with adjustable parameters}

A comparative analysis conducted on the basis of contemporary sources, leads to the conclusion that for effective dust cleaning is necessary to develop and use inertial dust hubs.

There is a need for further development of the scientific field and to create a universal equipment with greater efficiency, increased reliability and more features.

In order to implement our proposed destinations are encouraged to use the following technical solution, as reflected in the patent of the Russian Federation for useful model

(Fig. 1).

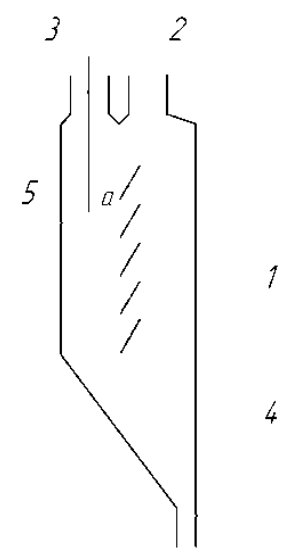

Fig. 1. Inertial dust hub: 1 - body, 2 - inlet pipe, 3 - output thin tube material, 4 - pipe the output of the coarse fraction, 5 - louver elements.

Inertial dust concentrator consists of a housing 1 . The upper housing inlet pipe 2 arranged starting material and output 3 fine material outlet. On the opposite side of the housing in its lower part located output nozzle 4 of the coarse fraction. In the middle part of the housing relative to its vertical axis mounted adjustable louver system elements 5 . Louvered fixed elements, such as axles, shafts, which are mounted on the side walls of the housing. Elements cinematically connected to each other, for example, traction, for simultaneous rotation about their axes and horizontal displacement. For this purpose, made in the body bore. Louvered elements are rotatable about their axes from $0^{\circ}$ to $180^{\circ}$ and move across the housing from $0 \mathrm{~mm}$ up to a.

Rotation is provided by the fact that on one of the axes, for example, the top mounted actuator or electro-mechanical or manual, and an axis between cinematically connected 
with the possibility of simultaneous rotation. Also, simultaneous movement of louver elements provided across the body from $0 \mathrm{~mm}$ to a. Varying quantities and parameters [alpha] and can widely alter the dispersibility of product exiting the dust concentrator. To control the process of separation of dispersed particles in a stream of air louver elements are rotated about their axes $0^{\circ}$ to $180^{\circ}$.

Inertial dust concentrator operates under discharge generated by an external fan. Dusty air enters the housing 1 on the hub inertial dust inlet pipe 2 and then passes to zone louver installation elements 5 . Due to the vacuum created by an external fan, the air flow with small turns the dust particles passing through $180^{\circ}$ louver elements and out through output pipe 3 of a dust concentrator. Large particles in the air stream by inertial forces trying to keep the trajectory of motion in a straight line in the output drop tube 4 and are removed from the hub.

Due to the fact that the louvre elements are able to rotate around their axes and to move horizontally, there are conditions for the control process of separating the dust-laden flow required particle size. You can also select such modes of his work in which, depending on the volume of pumping dusty air and dust can reduce the flow resistance of the device to a minimum.

This dust concentrator can be used in complex systems aerodynamic dedusting separation processes or fine powders, allowing the whole or improve dust collection efficiency of the separation.

\section{Strike the particles of the shoulder blade}

Upon impact, the spherical particles of the shoulder blade must change the center of mass of the particle velocity. Let the mass $m_{1}$ of a particle moving at a certain speed $V$, hits the vane mass $m_{2}$ at an angle [gamma] to the axis $x$. The surface of the blade body and consider it absolutely smooth.

The projections of the center of gravity of the speed of a falling body on the axis $\vec{n}$ and $\vec{\tau}$ (Fig. 2) are of the form:

$$
V_{n}=V \cos \delta, V_{\tau}=V \sin \delta
$$

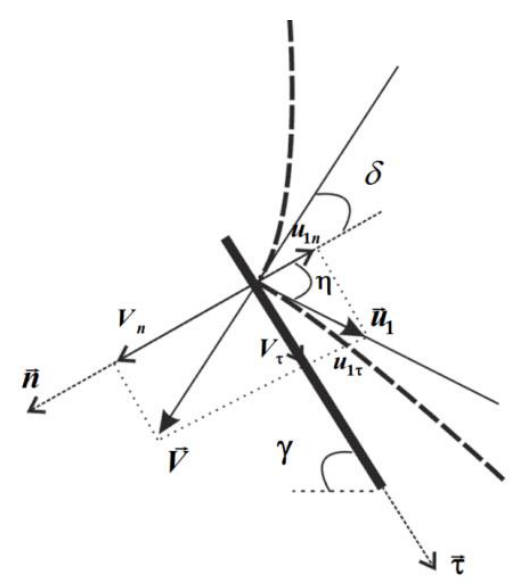

Fig. 2. Strike the particles of the shoulder blade to the hub. 
We consider an oblique partially elastic collision. Consideration of the impact carried out in two stages [8]. The first phase takes place a deformation of the colliding bodies. At the end of this phase velocity of the particle's center of gravity is given by

$$
u_{n}=\frac{m_{1} V_{n}}{m_{1}+m_{2}}=\frac{m_{1} / m_{2}}{m_{1} / m_{2}+1} V_{n} .
$$

This rate is the rate of inelastic collision.

During the second stage a partial recovery of non-deformed state. At the beginning of the second phase particle has the same speed as when the first closure. Projection particle velocity of the center of gravity at the end of the second stage (after impact) equal to

$$
\begin{gathered}
u_{1 \tau}=V_{\tau}=V \sin \delta \\
u_{1 n}=u_{n}+k\left(u_{n}-V_{n}\right)=\frac{m_{1} / m_{2}}{m_{1} / m_{2}+1} V \cos \delta+k\left(\frac{m_{1} / m_{2}}{m_{1} / m_{2}+1} V \cos \delta-V \cos \delta\right),
\end{gathered}
$$
written

where - coefficient of restitution $(0<k<1)$. After reduction of formula (4) can be

$$
u_{1 n}=u_{n}+k\left(u_{n}-V_{n}\right)=\frac{m_{1} / m_{2}-k}{m_{1} / m_{2}+1} V \cos \delta,
$$
(5)

Module of the particle velocity in the center of gravity in view of the end of a pin (3) -

$$
u_{1}=\sqrt{u_{1 \tau}^{2}+u_{1 n}^{2}}=\sqrt{(V \sin \delta)^{2}+\left(\frac{m_{1} / m_{2}-k}{m_{1} / m_{2}+1} V \cos \delta\right)^{2}} .
$$

Reflection of the particles will be at an angle $\eta$ [eta], the tangent of which is equal to

$$
\operatorname{tg} \eta=\left|\frac{u_{1 n}}{u_{1 \tau}}\right|=\left|\frac{V \sin \delta}{\frac{m_{1} / m_{2}-k}{m_{1} / m_{2}+1} V \cos \delta}\right|=\left|\frac{\operatorname{tg} \delta}{\frac{m_{1} / m_{2}-k}{\frac{m_{1} / m_{2}+1}{d}}}\right|=\left|\operatorname{tg} \delta \cdot \frac{m_{1} / m_{2}+1}{m_{1} / m_{2}-k}\right| .
$$

Because the particles are considered mass not exceeding $1.3 \times 10^{-5} \mathrm{~kg}$, it $m_{2}>m_{1}$. Then we can assume $m_{1} / m_{2} \approx 0$ and taking into account the conditions of the formula (5), (6) and (7) take the form

$$
\begin{gathered}
u_{1 n}=-k V \cos \delta, \\
u_{1}=\sqrt{V^{2} \sin \delta^{2}+k^{2} V^{2} \cos \delta^{2}},
\end{gathered}
$$




$$
\operatorname{tg} \eta=\left|\frac{\operatorname{tg} \delta}{-k}\right|
$$

In the case of an absolutely elastic impact $\mathrm{k}=1$ and

$$
\begin{gathered}
u_{1 n}=2 u_{n}-V_{n}=-V \cos \delta, \\
u_{1}=V, \\
\operatorname{tg} \eta=\operatorname{tg} \delta .
\end{gathered}
$$

These formulas make it possible to account for changes in the particle velocity vector in the reflection of the blades in the case of elastic or partially elastic impact.

\section{The particle deposition efficiency}

The acceleration of the vertical motion of the particles is determined by the resultant force of gravity, Archimedes and environmental resistance [9, 10]. In the case of gas Archimedes force can be neglected. Through elementary transformations [11], we obtain

$$
C_{X} \operatorname{Re}^{2}=\rho \frac{\left(\rho_{p}-\rho\right) d^{3}}{3 \mu \beta \frac{S_{p}}{V_{p}}} g
$$

How do we get the speed of the particles of hovering considering reflection particles from the blade (13)

$$
v_{B}=\frac{\left(\rho_{p}-\rho\right) d^{2}}{18 \mu \beta \frac{S_{p}}{V_{p}} \cos (\pi-2 \gamma)} g
$$

Then calculate the cleaning parameters $\mathrm{x} 1$ and $\mathrm{x} 2$ (partial parameters of the distribution function $\mathrm{F}(\mathrm{x})$ ) by the formulas [8]:

$$
x_{1}=\frac{H+h-L \frac{v_{B}}{v}}{\sqrt{2 D_{t}-\frac{l}{v}}}, \quad x_{2}=\frac{H-h-L \frac{v_{B}}{v}}{\sqrt{2 D_{t}-\frac{l}{v}}},
$$

where $\mathrm{h}$ - distance from the camera of the ceiling; $\mathrm{Dt}$ - coefficient of turbulent particle diffusion.

Next on the values of $x 1$ and $x 2$, we find the probability integral $\Phi(\mathrm{x} 1)$ and $\Phi(\mathrm{x} 2)$, and calculate the ratio of the concentration of particles in the size calculated at the outlet section of the chamber to their concentration in the inlet section $\mathrm{Ni}$

$$
N_{i}=\Phi\left(x_{1}\right)+\Phi\left(x_{2}\right)-1
$$

We average value of $\mathrm{N}$ in the cross section, calculating it as a simple average for the height of the section: 


$$
N_{a v}=\sum_{i=1}^{k} \frac{N_{i}}{k}
$$
size:

Next, we consider the average ratio of the partial deposition of the considered particle

$$
\varepsilon_{n . a v}=100-N_{a v}
$$

After this we find the full rate treatment as the sum of shares of the particles of the appropriate fractions to their fractional purification coefficients

$$
\varepsilon=\sum \varepsilon_{n} \frac{N}{100} \Delta d
$$

To validate the above expressions, we have performed a calculation of the concentrator with the following parameters: the volume of air aspirated $\mathrm{V}$ in inertial dust hub, width of a of the working area chamber, concentration [beta] of dusty air and the angle [alpha] tilt blade inertial dust hub.

Fig. 3 shows the influence of the volume of air aspirated V dust collecting efficiency $\varepsilon$ for different values of [beta] concentration of dust-laden air chamber working area of constant width of $\mathrm{a}=0.36 \mathrm{~m}$ and a tilt angle blades [alpha] $=40$ degree.

Fig. 3 shows that all the dependences are extreme character in the investigated range of varying factors.

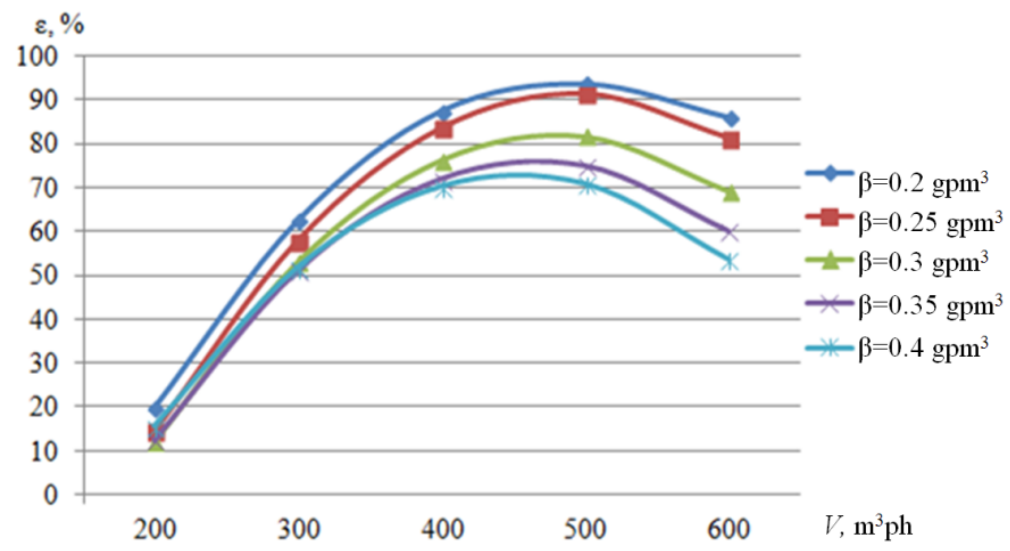

Fig. 3. Dependence of the efficiency of dust collection $\varepsilon$ aspirated air volume $V$ for various values of [beta] concentration of dust-laden air, a constant chamber width of the working area $\mathrm{a}=0.36 \mathrm{~m}$ and a tilt angle of the blades [alpha] $=40$ degree.

When analyzing the graphs shown in Fig. 3, it can be concluded that maximum dust separation efficiency is achieved when $\varepsilon$ aspirated air volume $\mathrm{V}=500 \mathrm{~m}^{3} \mathrm{ph}$, the concentration [beta] of dust-laden air [beta] $=0.2 \mathrm{gpm} 3$ and is [epsilon] $=93.6 \%$.

\section{Conclusions}

The conducted analyzes allowed to determine conditions conducive to increasing the efficiency of dust collection is due to changes in the design parameters, and by changing the process parameters, and also showed the need to clarify the experimental fractional composition of the output product. 
Improving the efficiency of dust collection inertial concentrator can be achieved both by varying the angle of inclination of the blades and due to increase to a certain value of air volume aspirated. Moreover, the increase in the volume aspirated air above a certain decline in the efficiency of dust collection entails.

As a result of experimental studies have provided updated according to the inertial dust concentrator, allowing to calculate the aerodynamic parameters of aspiration systems.

\section{References}

1. R.R. Sharapov, A.M Agarkov, World Applied Sciences Journal 24(10), 1399-1403 (2013)

2. N.S. Sevryugina, Construction and road machines 7, 28-29 (2007)

3. A.M Agarkov, Proceedings of the International scientific and technical conference. Kazan State Architectural University. Kazan., 7-11 (2015)

4. A.M Agarkov, L.M. Isanov, Y.I. Yalamov, The era of science 4, 89 (2015)

5. A.J. Waldberg. Theoretical foundations of air protection from pollution by industrial aerosols (MP "NIIOGAZ filter", Saint-Petersburg, 1993)

6. W.H. Duda. Cement-data-book. Band 1 - Internationale Verfahrenstechniken der Zementindustrie, Wiesbaden (Bauverlag, Berlin, 1985)

7. N.S. Sevryugina, V.M. Babin, Building and road machines 9, 49-53 (2007)

8. A.G. Vetoshkin. Processes and devices of dust cleaning. Tutorial (Penz. state University Press, Penza, 2005)

9. M.I. Bach, G.Y. Dzhanelidze, A.S.Kelzon. Theoretical Mechanics in examples and problems (Science, Moscow, 1973)

10. E.V. Kharlamov, R.R. Sharapov, M.A. Stepanov, Mechanization of construction 6(840), 28-32 (2014)

11. E.V. Kharlamov, R.R. Sharapov, V.G. Shaptala V.G., V.V. Shaptala, Bulletin of BSTU named after V. G. Shukhov 4, 91-95 (2013)

12. R.R. Sharapov, I.P. Boychuk, A.M Agarkov, V.S. Prokopenko, Bulletin of BSTU named after V. G. Shukhov 5, 175-178 (2015)

13. R.R. Sharapov, V.S. Prokopenko, A.M Agarkov, Bulletin of BSTU named after V. G. Shukhov 2, 84-89 (2015)

14. R. Schnatz, H.-G. Ellerbrock, S. Sprung, Zement-Kalk-Gips 48(2), 63-71 (1995) 